\title{
Nueva estrategia para la gestión basada en la confianza en organizaciones empoderadas
}

\section{Sumario}

1. Introducción

2. Resultados instrumentales disponibles para realizar decisiones colectivas

3. Mejoras que aporta la Web 2.0

4. ¿Cómo medir la empatía?

5. Nueva estrategia para decisiones conjuntas

6. Incorporación de la gestión de la confianza en las organizaciones

7. Discusión y conclusiones

8. Bibliografía

Fecha de entrada: 19-03-2017

Fecha de aceptación: 19-03-2018

Fecha de revisión: 05-04-2018

\section{Extracto:}

Este artículo propone una nueva estrategia para la gestión de organizaciones basada en la confianza. En las organizaciones empoderadas es posible realizar mediciones objetivas de la evolución de la confianza y, además, hacerlo automáticamente. La confianza es un proceso dinámico y su crecimiento o disminución es lo que aporta un significado real a las decisiones sociales. Esto inspira la estrategia propuesta: desarrollar procesos participativos mientras se produzcan aumentos de confianza y aplicar la mejor decisión obtenida hasta ese momento.

Los sistemas para medir la confianza se basan en la empatía entre los miembros de la organización, pero para su cálculo es necesaria una representación previa y homogénea de las opiniones de cada persona. La empatía entre todo par de individuos se mide a partir de la separación entre sus opiniones respectivas ante modificaciones sistemáticas en el plan de gestión de la organización. Posteriormente, se calcula la separación media. Los efectos de las interacciones entre personas se incorporan mediante modelos de simulación basados en agentes.

En cada caso de aplicación se debe determinar cuándo las mejoras marginales de confianza dejan de aportar mejoras significativas a la gestión de la organización. Para eso se adopta una regla de parada análoga a la desarrollada en los procesos de la implantación de sistemas de inteligencia artificial (IA).

Palabras clave: gestión de organizaciones, decisiones colectivas, empatía, sistemas complejos adaptativos y socialmente inteligentes, algoritmos estadísticos.

\footnotetext{
E. Martínez-Falero, catedrático de la Universidad Politécnica de Madrid, exdirector general de Industria, Energía y Minas de la Comunidad de Madrid, exviceconsejero de Economía y Empleo de la Comunidad de Madrid y expresidente de la Agencia de Acreditación de las Universidades de Madrid.

2 J. R. Peribáñez Recio, doctor ingeniero de Montes por la Universidad Politécnica de Madrid e inspector del Cuerpo de Bomberos de la Comunidad de Madrid (donde, además de sus funciones operativas de máxima jefatura en la atención directa a siniestros, ha sido responsable del Área Operativa y de Recursos Humanos) y egresado del Máster en Alta Dirección Pública.
} 


\section{New strategy for management based on confidence in}

\section{empowered organizations}

\section{Abstract:}

This paper proposes a new strategy for the management of organizations based on confidence. In empowered organizations, it is possible to make objective measurements of the evolution of trust and, in addition, to do it automatically. Confidence is a dynamic process and it is its growth or decrease that gives real meaning to social decisions. This inspires our strategy: to develop participatory processes while confidence increases, and then to apply the best decision obtained up to that moment.

The systems to measure confidence are based on the empathy among the members of the organization. But, it is necessary a previous homogeneous representation of any opinion. The empathy between each pairs of members is computed from the distance between their respective opinions to systematic changes in the management plan of the organization. The average separation is a global measure of empathy. We have incorporated the effects of member-to-member interactions through agent-based simulation models.

In each case of application, it must be determined when the marginal increases in confidence does not provide improvements to the management of the organization. The paper proposes a stop rule analogous to that developed for implementing artificial intelligence (Al) in organizations' management.

Keywords: organizational management, collective decision making, empathy, complex adaptive system socially intelligent, stochastic algorithms.

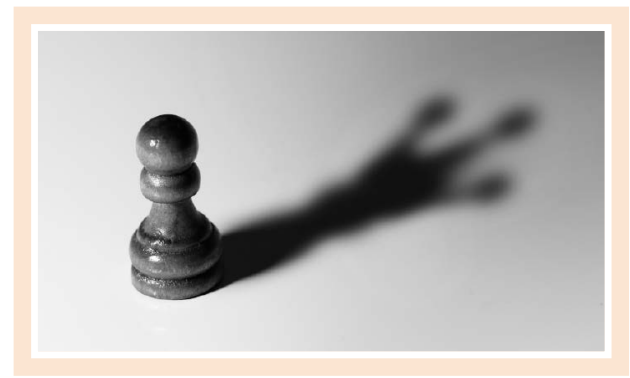

\section{INTRODUCCIÓN}

¿Qué pasaría si nos fiáramos de los demás al tomar nuestras propias decisiones?

La presencia de un número elevado de decisores mejora las decisiones individuales. Al procesar, a través de la web, información de muchos participantes, se mitigan los sesgos negativos que se producen en las decisiones individuales (Myers, 2002).

Además, cuando se trabaja en entornos de confianza, las opiniones individuales suelen converger. En estos entornos, cada persona toma en consideración las razones de los otros. Para eso, en primer lugar, debe conocerlas (verlas reflejadas explícitamente -como ocurre en los procesos participativos-) y, en su caso, reformular los juicios personales a la luz de esa información. Por tanto, las opiniones convergen cuando se produce un aumento de empatía entre los componentes de la organización. La empatía se refuerza con el uso de un lenguaje común de comunicación (las tecnologías de la información y la comunicación [TIC]) y cuando los individuos se han formado en criterios éticos y de justicia compartidos.

\section{La presencia de un número elevado de decisores mejora las decisiones individuales. Al procesar, a través de la web, información de muchos participantes, se mitigan los sesgos negativos que se producen en las decisiones individuales}


Pero las decisiones conjuntas no se pueden imponer: nada hace avanzar más a una sociedad que la capacidad de asumir riesgos personales, lo que es inseparable del derecho de que cada uno disponga de los beneficios que se obtiene de la exposición al riesgo.
En la gestión de las organizaciones se puede aprovechar lo mejor de ambas posiciones: dar poder a los individuos en los asuntos en que son expertos y mantener los objetivos y procedimientos generales sujetos a la política general de la organización.

\section{RESULTADOS INSTRUMENTALES DISPONIBLES PARA REALIZAR DECISIONES COLECTIVAS}

Aunque no sea posible asegurar que se vayan a conseguir decisiones que resulten lo suficientemente buenas para todos, sí se pueden obtener soluciones que tengan en cuenta la utilidad personal y que sean socialmente aceptables.

Esto no sucedía en los primeros modelos para desarrollar soluciones conjuntas que se basaban en formalizar estrategias para juegos en red. En estos modelos, cada agente trata de maximizar su ganancia personal, lo que conduce a una reducción en las ganancias de los demás. Para la resolución de estos conflictos fue necesario incorporar algún tipo de colaboración social, por lo que la generación de confianza tomó carta de naturaleza. En todo caso, aunque el énfasis se ponga en alguno de los extremos, es evidente que los actuales sistemas para decisiones colectivas tienen en cuenta tanto las preferencias personales como las interacciones sociales y se ajustan mejor al punto de vista de toda la población.

Todas las aplicaciones informáticas disponibles para facilitar el desarrollo de procesos participativos se basan en alguno de los modelos anteriores. Todas ellas tienen importantes campos de mejora, pero aportan soluciones operativas. A continuación, se presentan las principales aplicaciones para decisiones participativas.

\subsection{Aplicaciones tipo $A$}

Los primeros modelos para desarrollar soluciones conjuntas se basaron en formalizar estrategias para juegos en red. En estos modelos se distinguen dos tipos de procedimientos:

- Sistemas de apoyo a las decisiones en grupo (GDSS). Pretenden llegar a una decisión negociada global entre los participantes a través de reuniones presenciales.

\section{[...] los actuales sistemas \\ para decisiones colectivas \\ tienen en cuenta tanto las \\ preferencias personales como \\ las interacciones sociales y se \\ ajustan mejor al punto de vista \\ de toda la población}

- Sistemas de apoyo a las decisiones sociales (SDSS). Visualizan el flujo de la discusión a través de una red de declaraciones, opiniones, argumentos y comentarios, que ayuda a obtener un consenso antes de votar sobre una cuestión.

\section{A) Aplicaciones tipo A1 (GDSS)}

Entre los muchos programas disponibles destacan los siguientes:

- PLEXSYS. De la Universidad de Arizona.

- Colab. Diseñado en Xerox en el Centro de Investigaciones de Palo Alto.

- DECAID (decision aids for groups). Diseñado por Gallupe, DeSanctis y Dickson.

- LADN (local area decision network).

- SMU (Southern Methodist University).

- Loomio. Es el programa más utilizado (usado en España en la toma de decisiones de los círculos del partido Podemos). 


\section{B) Aplicaciones tipo A2 (SDSS)}

Las aplicaciones de este tipo más utilizadas son:

\section{- INCOMA.}

- Shell SDSS. Fue desarrollado en la Universidad de Minnesota.

- Deliverative Opinion Poll. Desarrollado en la Universidad de Stanford.

\subsection{Aplicaciones tipo B}

En el ámbito de la colaboración social, los sistemas disponibles implementan modelos que simulan la propagación de opiniones, como se hace con los procesos de infección o contagio. De hecho, los agentes sociales y ambientales han buscado siempre identificar los procesos de colaboración social para conocer la propagación de sus opiniones entre los componentes de la sociedad.

El software disponible para simular estos modelos no es estrictamente de toma de decisiones colectivas, sino de análisis y tratamiento de la información (estructurada y no estructurada), con múltiples receptores. No obstante, algunas aplicaciones han evolucionado hasta presentar una solución agregada. Por la focalización que ponen en diferentes aspectos, se distinguen aplicaciones que priorizan la organización de la información (Paramount Decisions, Analytica, etc.), la evaluación por múltiples agentes (1000Minds, de IBM) y la aplicación de procedimientos para predecir la evolución de sistemas complejos (el análisis de sistemas dinámicos y las simulaciones del comportamiento de los agentes del sistema).

\subsection{Aplicaciones tipo C}

Las aplicaciones referidas a los sistemas que tienen en cuenta tanto las preferencias personales como las interacciones sociales conducen a soluciones eficaces y no siempre son eficientes. Se destacan dos programas por su gran utilización:

- LiquidFeedback. Esta aplicación, desarrollada en el Massachusetts Institute of Technology (MIT), incorpora redes sociales de confianza de forma que se puede delegar la decisión en otros (democracia líquida). No realiza preguntas directas sobre el resultado final, sino que alienta a los participantes a proponer alternativas. Dispone de un sofisticado sistema de votación para permitir a los participantes expresar sus opiniones sin consideraciones tácticas.

- All Our Ideas. Usa un método de comparación entre pares de alternativas que debe seleccionar cada usuario para ordenar una lista de propuestas (a la que pueden añadirse otras nuevas). Con la información de los usuarios se elabora un ranking de las propuestas preferidas por todo el grupo. Esta aplicación se ha desarrollado en la Universidad de Princeton.

\section{MEJORAS QUE APORTALA WEB 2.0}

Las mejoras que aportan las soluciones conjuntas se consiguen al incidir en tres aspectos (Bonabeau, 2009):

- Alcance entre los posibles participantes.

- Agregación de opiniones diferentes para posibilitar la convergencia a una solución socialmente inteligente.

- Autoorganización.

\subsection{Aumento del alcance}

El alcance se refiere al número de participantes en las decisiones colectivas, porque no solo es un hecho que nadie lo sabe todo, sino que también sucede que no hay nadie que no sepa nada y no pueda aportar algún conocimiento útil. El teorema de Condorcet (1785) resume este aspecto: si los componentes de un grupo están bien informados y el número de participantes crece hasta incorporar a todos los individuos del grupo, entonces, el grupo, con certeza, tomará decisiones acertadas (aunque, también, al contrario: si crece el número de individuos mal informados de un grupo, el grupo, con certeza, errará en sus decisiones).

La mayor dificultad para la aplicación del teorema de Condorcet es la participación plena: a medida que los grupos crecen en tamaño no se puede esperar una participación de todos en todas las decisiones. Pero los no participantes pueden delegar su voto en los participantes activos más cercanos a ellos en las redes sociales y transmitir el derecho a tomar decisiones a través de una red social basada en la confianza (formalmente, la confianza que deposita una persona en 
cualquier otra es la probabilidad subjetiva de que sintonicen sus opiniones). Otra dificultad para aplicar el teorema de Condorcet es que todos los participantes estén bien informados, $y$, aunque este hecho no se puede asegurar, es posible actuar para aumentar la probabilidad de que esto ocurra (a través de la extensión de la educación humanística y de la difusión del conocimiento científico $)^{3}$.

\subsection{Agregación de opiniones}

Cuando muchas personas emiten sus juicios para evaluar una solución, hay tantas personas que la sobrevaloran como tantas que la infravaloran. El resultado es que el promedio de evaluaciones se acerca a la evaluación real. Sin embargo, no todos tienen por qué aceptar esa solución promedio.

Los primeros trabajos para alcanzar soluciones consensuadas se centraron en el concepto de «elección social», que relacionaba las preferencias sociales e individuales mediante una función de bienestar social (Borda, 1784; Condorcet, 1785). El principal instrumento de elección social es la votación ${ }^{4}$, aunque la mayoría de las aplicaciones desarrolladas para la Web $2.0^{5}$ también se pueden considerar elecciones sociales. Por desgracia, no se puede afirmar que ni siquiera las condiciones más simples de razonabilidad se vayan a cum-

\footnotetext{
${ }^{3}$ En cualquier caso, existen algoritmos que facilitan el proceso de representación social. Entre los modelos más usados se encuentran los dinámicos, que utilizan grafos aleatorios para representar los flujos de opinión entre participantes no activos y representantes activos en los que confian influir con sus opiniones (Grönlund, Holme y Minnhagen, 2007). Otros modelos incorporan la probabilidad subjetiva de que dos agentes sintonicen sus opiniones (valoraciones, racionalidad y profundidad de conocimiento) (Rodríguez et al., 2007) y elementos de la lógica difusa en los gráficos que representan las redes sociales basadas en la confianza para la toma de decisiones colectivas (Rovarini, Cerviño y Juárez, 2009).

${ }^{4}$ El voto es la forma natural de expresar opiniones en democracia. Hay muchos sistemas disponibles -véase, por ejemplo, Stensholt (2011)-, aunque no todos son inocuos. Así, los métodos que expresan preferencias permiten que, mediante estrategias adecuadas, los resultados puedan ser manipulados para garantizar, a priori, la elección de algunas alternativas. Por otra parte, la votación mayoritaria no permite considerar las opiniones de las minorias.

${ }^{5}$ Las principales soluciones que aporta la Web 2.0 son la ordenación de documentos, la folksonomy (una clasificación colaborativa por medio de etiquetas simples en un espacio plano y sin jerarquías ni asociaciones predeterminadas), los sistemas recomendadores, las wikis, el software colaborativo de código abierto, la propia votación y los mercados electrónicos de predicción.
}

plir siempre y simultáneamente en todos los procesos de elección social. De hecho, la agregación de las preferencias por elección social requiere eludir el teorema de imposibilidad de Arrow (1963) mediante la relajación de algunas de sus condiciones.

Poco después de surgir el concepto de «elección social», Bentham (1789) introdujo la noción de «utilidad», interpretada como la satisfacción -el equilibrio de placer y de dolor- que una persona experimenta por la adquisición de un bien. Esta noción, retomada por su editor, Stuart Mill, se convirtió en el criterio fundamental para la toma de decisiones. Los utilitaristas (Edgeworth, 1881; Marshall, 1890) emplearon la suma de las utilidades individuales para obtener evaluaciones sociales, si bien su preocupación era la utilidad total de la comunidad (y no la forma en que se distribuía o se concentraba).

No obstante, a partir del positivismo lógico (Robbins, 1938) se incorporaron criterios de promoción social y nuevas funciones de bienestar (igualitarismo, eliminación de la envidia, gratuidad, etc.). Este hecho facilitó la identificación de las pérdidas y de las ganancias individuales y permitió avanzar hacia la realización de comparaciones interpersonales de utilidades ${ }^{6}$. Junto al positivismo, el avance de la «revolución marginalista», que posibilitaba no atribuir utilidades a los agentes para demostrar la mayoría de los postulados económicos, terminó por arrinconar casi todo el bagaje de Bentham.

Von Neumann y Morgenstern (1944) propusieron medir el bienestar de una persona a partir de la utilidad marginal decreciente (o creciente) del dinero. La utilidad que proponen estos autores se mide por el riesgo que está dispuesto a asumir una persona para obtener un bien ${ }^{7}$. Esta teoría, además, se puede extender al cálculo de utilidades interpersonales mediante la agregación de suposiciones adicionales basadas en

\footnotetext{
${ }^{6}$ Pero ¿es posible realizar comparaciones entre los tamaños de las utilidades de dos o más personas? Indudablemente ha habido progresos en este sentido: se han realizado comparaciones interpersonales de utilidad a partir de diferentes principios -véase, por ejemplo, Rawls (1971)-y se han definido condiciones muy generales para su implementación (Hammond, 1991).

${ }^{7}$ Los supuestos de racionalidad incorporados en la teoría de Von Neumann simplemente requieren que las personas tomen decisiones de manera consistente, pero sus conclusiones son sorprendentemente fuertes. Debido a que una persona racional actúa como si maximizara la utilidad esperada o promedio, se comporta como si cada unidad de utilidad de cualquier bien «valiera» lo mismo que la de cualquier otro.
} 
la empatía entre los componentes de una organización (Harsanyi, 1955, 1977 y 1992) ${ }^{8}$.

En la actualidad, se incide más en una teoría descriptiva del comportamiento que en teorías explicativas del mismo ${ }^{9}$. A partir de la descripción, se puede saber si una persona actuará de manera más o menos consistente por su forma de proceder en el pasado. La descripción hunde sus raíces en el comportamiento observado; por tanto, esta teoría se denomina «teoría de las preferencias reveladas ${ }^{10}$.

Como se verá posteriormente, el estado actual de las TIC permite incorporar fácilmente y procesar las preferencias reveladas, incluyendo la noción de «empatía» (puesto que a partir de las preferencias reveladas no se tiene dificultad para describir el comportamiento empático de una persona respecto de otra).

\subsection{Autoorganización}

Se trata del proceso en que la coordinación surge de las interacciones entre los miembros de una organización. La autoorganización potencia la aparición de fenómenos emergentes (soluciones diferentes a la mera agregación que hace que el todo sea más que la suma de sus partes).

\footnotetext{
8 Suppes (1966) introdujo estas preferencias que, después, fueron estudiadas por Sen (1970 y 1976) y Arrow (1978) bajo el nombre de «preferencias de simpatía extendidas». Aunque ya Smith (1759), siguiendo a Hume (1739), consideró la simpatía en su teoría de los sentimientos morales, es evidente que los filósofos morales posteriores solo apelaron a la simpatía cuando necesitaron de algún apoyo auxiliar para llegar a una conclusión (Hardin, 1988). De hecho, hasta que no apareció la teoría de juegos, no hubo herramientas disponibles para poner en práctica las ideas de Hume.

En todo caso, hay que remarcar que lo que Hume y Smith llamaron «simpatía», hoy en día se conoce como «empatía». Los psicólogos reservan la palabra «simpatía» para una noción más fuerte. Para nuestro objetivo, una persona $A$ simpatiza o empatiza con otra $B$ cuando se imagina a s mismo en los zapatos de B, para ver las cosas desde su punto de vista.

9 Es cierto que la teoría de Von Neumann y Morgenstern no ha satisfecho a todos y que las ideas del enfoque empírico de Bentham han revivido a través de los «estudios de la felicidad» (Layard, 2005). Incluso los neurocientíficos están dispuestos a contemplar la posibilidad de que algún dispositivo de medición pueda conectarse al cerebro para medir cuánto placer o dolor experimenta una persona. No obstante, la mayoría de los economistas ya no se aferran a las creencias sobre el funcionamiento de nuestras mentes y abandonan cualquier intento de explicar por qué las personas se comportan como lo hacen.

10 La utilidad de que un bien $(X)$ sea mayor que la de otro $(Y)[u(X)>u(Y)]$ solo se debe a que se ha revelado la preferencia $X>Y$.
}

En lugar de eliminar la aleatoriedad de la vida, la autoorganización facilita que cada persona revise sus decisiones a cada paso en el proceso dinámico de prueba y error que conforma toda actuación personal. En realidad, el elemento aleatorio no lo es tanto, porque cada fallo detiene una línea de actuación e imposibilita cometer errores mayores en el futuro en esa dirección. Incluso cuando una persona toma una decisión que le perjudica irreversiblemente, su error favorece a toda la comunidad cuando los demás aprenden de él (esto sucede en la evolución natural, pero también con la tecnología y la computación).

Pero la autoorganización solo impulsa el progreso social cuando se realiza en entornos de confianza: de autoconfianza de los individuos que se arriesgan a implantar nuevas soluciones, de confianza en que los que no se hayan expuesto al riesgo no se puedan apropiar de los beneficios de los emprendedores (problemas éticos que transfieren fragilidad al sistema [Taleb, 2012]) y de confianza en que la comunicación de los éxitos llega a todos en igualdad de oportunidades (lo que se ve facilitado por el uso de las TIC). Además, en entornos de identificación empática (entornos de confianza) se puede esperar que la aplicación de convenciones sociales a una posición original acelere el camino hacia el equilibrio respecto de los métodos más lentos de prueba y error (Binmore, 2005).

\section{4. ¿CÓMO MEDIR LA EMPATÍA?}

El actual desarrollo de las TIC posibilita un conocimiento sin parangón del funcionamiento de las organizaciones: del desempeño de sus componentes, de sus interacciones y de cómo aprenden y se adaptan a situaciones cambiantes.

Al incorporar las decisiones personales en los sistemas de información, las TIC pueden identificar automáticamente las preferencias de cada persona ( 0 grupo) y representarlas. Esto se realiza a partir de su comportamiento histórico -preferencias reveladas en el desempeño del trabajo o como consumidor- y de la forma en que cada uno justifica las decisiones tomadas (básicamente a partir del significado que atribuye a la interacción entre las características y cualidades de las posibles soluciones). 


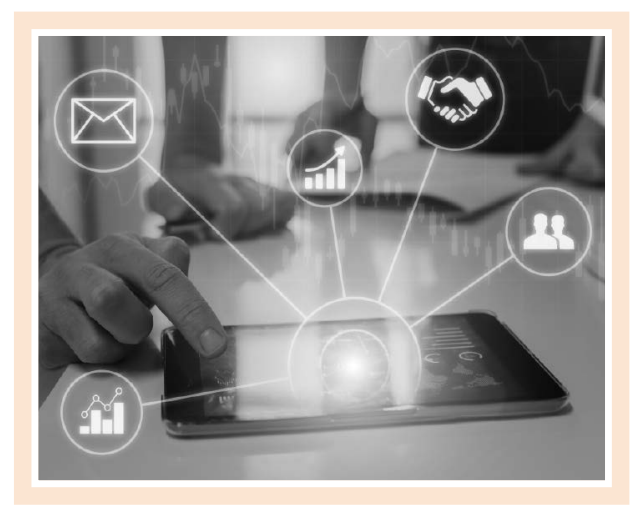

Cuando se dispone de la representación de las preferencias individuales es posible valorar cualquier solución según lo haría la persona cuyas preferencias se han representado. Además, si la representación de preferencias fuera homogénea, se podrían realizar comparaciones de las valoraciones de distintos individuos.

La valoración también permite diseñar la alternativa que mejor se adapta a las preferencias identificadas y establecer comunidades de personas con los mismos sistemas de preferencias.

Específicamente, la opinión se describe por la forma en que se valora cualquier solución y por la identificación de la solución que se considera mejor (véase epígrafe 4.1).

Pero las TIC no solo permiten describir opiniones automáticamente. El estado actual de su evolución posibilita la agregación de opiniones a partir de la teoría de Harsanyi (1977 y 1992), que añade la noción de empatía a las hipótesis generales sobre utilidad de Von Neumann y Morgenstern.

Si se acepta que la empatía supone ver las cosas desde el punto de vista del otro, es fácil entender por qué la evolución biológica puede llevarnos a comportarnos como si dispusiéramos de preferencias empáticas (Page y Martin, 2002). De hecho, las madres suelen cuidar a sus hijos más que a sí mismas. Pero los humanos también empatizamos con los esposos y esposas, con las familias extendidas, con los amigos y vecinos y con la secta o la tribu. En estas condiciones, la teoría de Von Neumann y Morgenstern es suficiente para determinar un estándar de comparación interper- sonal (porque una persona solo necesitaría consultar su propia función de utilidad empática para averiguar cuántas utilidades asignar a un cambio en la situación de otra persona, en comparación con cambios que se produjeran en su propia situación).

Sin embargo, el enfoque de Harsanyi es más completo. Junto con nuestras preferencias personales, que pueden incluir o no preocupaciones comprensivas para otros, también empatizamos con la ética de nuestras propias actuaciones. De hecho, cuando nos identificamos con otra persona, no lo hacemos hasta el punto de no poder separar nuestras preferencias de las suyas (todos podemos ponernos en el lugar de una persona que está enferma, pero, en el caso de que la persona enferma fallezca, no necesariamente querríamos morir también). Otro requerimiento de Harsanyi es la idealización de su modelo de decisor, al que asigna preferencias empáticas consistentes con objeto de satisfacer los requisitos de racionalidad de Von Neumann y Morgenstern (véase nota 7).

Para la determinación de la empatía hay que incorporar las preferencias altruistas a las preferencias personales. Con estos dos tipos de preferencias, cualquier persona solo tendría que consultar su propia función de utilidad empática para averiguar cuántas unidades de su utilidad debe asignar a cualquier modificación en una solución, de forma que ese mismo cambio en otra persona sea comparable al que se produce en su propia situación.

En consecuencia, las preferencias empáticas deben distinguirse de las preferencias personales integradas en la función de utilidad personal11 ${ }^{11}$. Una función de utilidad personal ortodoxa, del tipo de las que se desarrollan en el epígrafe 4.1, asigna una utilidad a cada situación que la persona en cuestión pueda encontrar.

\footnotetext{
11 Binmore presenta un interesante ejemplo de esta separación cuando expresa sus preferencias empáticas en el conflicto entre Adán y Eva: «[...] cuando digo que preferiría ser Eva comiendo una manzana que Adán con una hoja de parra, mis propias preferencias personales son irrelevantes [...]. Como yo no soy un chico de la playa, personalmente preferiría una hoja de parra para cubrir mi desnudez que una manzana para agregar a mi cintura. Sin embargo, como sé que las manzanas tienen un sabor muy dulce para Eva y que Adán es totalmente inconsciente de su cuerpo, claramente no empatizaría con éxito si mis propios impulsos hacia la modestia influyeran en mi juicio sobre si Eva está obteniendo más satisfacción de su manzana que la que Adán obtiene de su hoja de parra».
} 
En cambio, para construir una función de utilidad empática, debemos vincular cada situación con la persona a la que estamos considerando en esa situación. Una posible vinculación procedería de analizar los posibles pares formados por un operario procesando un problema de una forma y otro procesándolo de otra forma. La función de utilidad empática asignaría una utilidad a cada par $^{12}$ (véase epígrafe 4.2).

La medida de las empatías recíprocas entre dos individuos indica el grado de convergencia de sus opiniones explícitas a una opinión conjunta. El promedio de las empatías para todos los pares de evaluadores es un indicador de la empatía de toda la organización y es la medida adoptada para evaluar la confianza en toda la organización ${ }^{13}$.

A partir de los sistemas de información es posible monitorizar automáticamente los cambios de empatía a lo largo del tiempo y conocer los cambios de confianza ante variaciones en la gestión de la organización. Las soluciones que aporten mejoras a los procesos actuales y que, al mismo tiempo, supongan un aumento de confianza global serán decisiones aceptables para la organización (serían socialmente aceptables si la organización fuera toda la sociedad).

\subsection{Representación de la opinión}

La representación de la opinión de cualquier persona requiere conocer cómo valora cada individuo cualquier alternativa de solución y cuál es la mejor solución para él.

Pero la valoración no es un problema simple: las posibles soluciones a los problemas sociales se describen a través de múltiples variables (una solución tiene, por ejemplo, un coste, una tasa de rendimiento, genera un número de puestos de trabajo, etc.). Esto dificulta la aplicación de una lógica de comparación de soluciones aceptada universalmente: ¿qué es mejor,

\footnotetext{
12 Estos son los pares de posibilidades que deben evaluarse cuando las personas se imaginan en la "posición original» detrás de un «velo de ignorancia» que oculta hipotéticamente la identidad de cada individuo.

13 Es evidente que el promedio de empatías entre pares de participantes no describe la distribución de la confianza en una organización (es imprescindible apoyarse en otras medidas de centralización y de dispersión para un análisis formal de la confianza), pero no olvidemos que nuestro objetivo es medir la evolución de la confianza, y la variación del promedio de empatía en el tiempo permite detectar los crecimientos o las disminuciones de empatía total.
}

un proyecto que cuesta 2.000 .000 de euros y obtiene 5.000 .000 de euros u otro que cuesta 500.000 euros y obtiene 2.000 .000 de euros? No hay una respuesta aceptable para todos, la valoración de alternativas con múltiples prestaciones (que requieren múltiples variables para describirlas) es propia de cada individuo y se deduce de patrones personales de comparación. La valoración transforma las preferencias (es decir, la opinión) de cada persona en un valor numérico.

El valor de una alternativa es un número que permite saber cómo valora cualquier persona cualquier situación. Cuando la valoración es homogénea, permite, además, que terceros realicen comparaciones de las preferencias de cada individuo con las de otros.

Desde un punto de vista metodológico hay tres procedimientos para valorar opiniones individuales:

- Valoración a partir de comparaciones de pares de alternativas -modelización de preferencias- (véase Öztürk, Tsoukiàs y Vincke [2005] para una descripción detallada del estado del arte de esta metodología).

- Cálculo de la utilidad a partir de las interacciones entre las prestaciones que caracterizan las alternativas (formalizado en primer lugar por Debreu, 1954).

- Asignación de valor por análisis de las decisiones pasadas (desarrollado a partir de los trabajos de Arrow, 1959).

Los resultados que se derivan de la aplicación de los tres procedimientos anteriores son intercambiables (Aleskerov, Bouyssou y Monjardet, 2007), y, así, entre otros, los trabajos de Suzumura (1983) y de Sen (1987) permiten trasponer los conceptos de "preferencias», «utilidad» y «elecciones» entre sí y manejar, indistintamente, las capacidades de cálculo de cualquiera de las mencionadas metodologías (véase, por ejemplo, Martínez-Falero, Martín-Fernández y Orol [2013], MartínezFalero, Ayuga-Téllez, González-García, Grande-Ortiz y Sánchez de Medina [2017] y Liu y Teng [2016] para consultar un procedimiento instrumental de valoración de utilidades personales).

La valoración también facilita obtener la alternativa que mejor se adapta al sistema de preferencias de cualquier evaluador, sea este un evaluador individual o un grupo, aunque, en general, el número de posibles soluciones a los problemas sociales es tan elevado que no suele ser posible generar todas las alternativas 
de solución y evaluarlas para identificar la mejor. En su defecto hay que utilizar herramientas de optimización combinatoria para la obtención de la mejor solución.

Alternativamente, se suele determinar la mejor solución en condiciones de riesgo y adoptar una solución con alta probabilidad de satisfacer un determinado sistema de preferencias (lo que en muchos casos es suficientemente bueno). La probabilidad de que una alternativa sea la mejor también se puede construir a partir de la valoración de alternativas.

Como sucede con la valoración, la búsqueda de la «Solución aceptable» también está resuelta metodológicamente. En este caso, se realiza mediante la aplicación de algoritmos recursivos; de algoritmos de optimización heurística (programación matemática, métodos Montecarlo; algoritmos genéticos; simulaciones de sistemas naturales, búsqueda tabú, etc.) y de algoritmos de optimización no-heurística (básicamente el análisis de redes neuronales); 0 a través de una combinación de los tres tipos de algoritmos anteriores (véase, por ejemplo, Martín-Fernández, Martínez-Falero y Valentín-Gamazo [2013] y MartínFernández y Martínez-Falero [2018] para consultar un procedimiento instrumental de optimización combinatoria).

\subsection{Cálculo de la empatía}

Como se verá en epígrafes posteriores, la evaluación de la empatía se basa tanto en la algorítmica como en la relación entre datos y algoritmos. Los algoritmos no solo deben "aprender» de los datos, sino que también han de adaptarse a los mismos, hasta el punto de aplicar procedimientos específicos para cada caso.

A continuación se describe un procedimiento general, válido en casos con un elevado número de evaluadores y múltiples soluciones posibles (aunque finitas) para cada evaluador. Se basa en calcular la empatía a partir de evaluadores conectados en una red de confianza (Salehi-Abari y Boutilier, 2014) e incorpora las últimas aportaciones a la teoría de Harsanyi, desarrolladas por Adler (2014), si bien no se ha adoptado la notación terminológica de este autor, sino la desarrollada en la primera aplicación del algoritmo propuesto (Ayuga-Téllez, González-García y Martínez-Falero, 2013), que medía la empatía en un proceso de partición para la gestión de un espacio público.
Parece evidente que cuando se quiere determinar la empatía de una persona $(A)$ hacia otra $(B)$, hay que considerar las opiniones de todos aquellos que conforman la opinión que $A$ cree que $B$ tiene de cualquier solución $X$. Como mínimo serán las opiniones de $A$ y de $B$, las de las personas que $A$ considera que están más próximas a $B$ en las redes de confianza $n(B)_{A}$, el histórico de las diferentes opiniones agregadas a lo largo del proceso participativo, 0 , al menos, la evaluación agregada de todo al grupo en el momento en que se calcula la empatía $(g)$-que se identifica con la posición original- y, con el fin de promover la imparcialidad en la valoración final, una opinión explícita objetiva formulada por expertos $(\mathrm{Ob})$.

A partir de las opiniones que conforman la empatía se puede determinar la utilidad que una persona $(A)$ cree que una solución cualquiera $X$ tiene para otra persona $(B)$.

Pero no todas las opiniones tienen la misma importancia. Así, la utilidad que $A$ cree que la solución $X$ tiene para $B$ se calcula como:

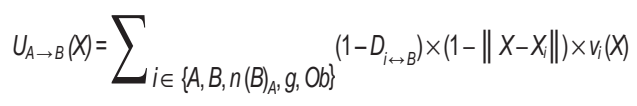

Donde $D_{i \leftrightarrow B}$ es la separación entre las opiniones de $i$ y de $B$ en las redes de confianza; $\left\|X-X_{i}\right\|$ es la distancia entre la mejor solución para $i\left(X_{j}\right)$ y la solución para la que calcula la utilidad $(X) ; v_{i}(X)$ es la evaluación que $i$ hace de $X$; finalmente, $i$ se refiere a las opiniones involucradas:

$$
i \in\left\{A, B, n(B)_{A}, g, O b\right\}
$$

En todo caso, la forma en que $A$ cree que $B$ valora cualquier solución $X$ no es todavía la función de empatía de $A$ respecto de $B$. La empatía expresa la equivalencia en las utilidades de $A$ y $B$ cuando se modifica la evaluación agregada del grupo. Así, la empatía de $A$ con respecto a $B$ es un vector de $L$ componentes que refleja cómo $A$ cree que $B$ valoraría $L$ modificaciones sistemáticas en la solución que se considera la mejor para todo el grupo $\left(X_{g}\right)$. El conjunto de funciones de empatía de $A$ para todos los evaluadores de una organización se puede representar por una matriz cuyas filas son los vectores de $L$ componentes antes aludidos. 
Si, como antes, se representa por $U_{A \rightarrow B}(X)$ la utilidad que $A$ cree que una solución cualquiera $(X)$ tiene para $B$, para el cálculo de la empatía de $A$ respecto de $B$ se parte de la mejor solución para el grupo $\left(X_{g}\right)$ y se provocan $L$ cambios sistemáticos en los valores de sus variables descriptoras (por ejemplo, aumentando o disminuyendo el coste y el rendimiento de $X_{g}$ ). Cada uno de esos cambios lo representaremos por $X_{g<k>}$ (para $k=1, \ldots, L$ ). Las modificaciones que esos cambios producen en la forma en que $A$ cree que $B$ realiza sus valoraciones de utilidad serán:

$$
e_{A \rightarrow B, k}=U_{A \rightarrow B}\left(X_{g<k>}\right)-U_{A \rightarrow B}\left(X_{g}\right)
$$

Para $k=1, \ldots, L$ se obtiene un vector de $L$ coordenadas que ya representa la empatía de $A$ respecto de $B$. El conjunto de las empáticas de $A$ para todos los $N$ evaluadores será una matriz que representaremos como euf $A_{A}$.

$$
\operatorname{euf}_{A}=\left[\begin{array}{ccc}
e_{A \rightarrow \rightarrow A_{1}, 1} & \cdots & e_{A \cdots \rightarrow A_{N}, 1} \\
\vdots & \ddots & \vdots \\
e_{A \rightarrow A_{1}, L} & \cdots & e_{A \rightarrow A_{N}, L}
\end{array}\right]
$$

Dado que la matriz que describe la empatía de una persona con el resto de evaluadores es una tabla de $N$ filas (si hubiera $N$ evaluadores) y $L$ columnas (que muestran la variación de utilidades a $L$ cambios en la solución agregada), a partir de operaciones generales con matrices, se puede obtener una medida de la separación de empatías entre cualquier par de indivi$\operatorname{duos}\left(S_{A B}\right)$.

En general, la separación de empatías entre todo par de individuos se puede expresar como:

$$
S_{A B}=1-\left(\left\|\operatorname{euf}_{A}, \operatorname{euf}_{B}\right\| / N\right)
$$

Donde:

- N es el número de evaluadores.

- $\| \operatorname{euf}_{A}$, euf $_{B} \|=\sqrt{\operatorname{tr}\left[\left(\operatorname{euf}_{A}-\operatorname{euf}_{B}\right)^{\circ}\left(\operatorname{euf}_{A}-\operatorname{euf}_{B}\right)^{T}\right]}$.

- o representa el producto de matrices.

- $\operatorname{tr}()$ es la traza de una matriz.

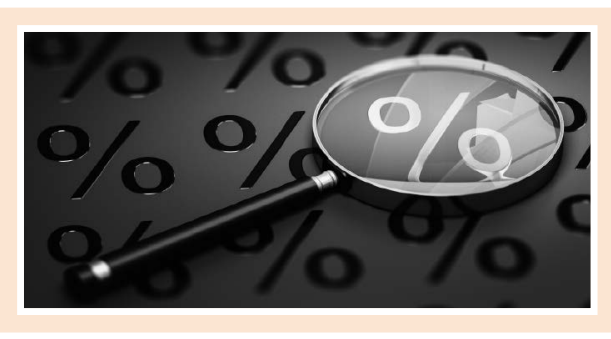

El promedio de empatías entre todo par de evaluadores es la medida de la empatía para la organización (véase nota 13):

$$
S_{T}=\frac{1}{N(N-1) / 2} \sum_{l=1}^{N-1} \sum_{J=l}^{N} S_{l J}
$$

Pero más importante que el cálculo de un indicador es considerar la empatía como un proceso dinámico en el que los evaluadores interactúan entre ellos y con otros agentes. En un proceso participativo real, la expresión anterior se modificará a lo largo del proceso; pero también se puede conocer el efecto de las interacciones a priori, mediante la aplicación de modelos de simulación basados en agentes (Dionne, Sayana, Hao y Bush, 2010; Sayama, Farrell y Dionne, 2011; Ayuga-Téllez, González-García y Martínez-Falero, 2013; McHuga, Yammarino, Dionne, Seban, Sayama y Chatterjee, 2016). La aplicación de la metodología expuesta a un caso real de participación para determinar el mejor uso de un espacio público ha supuesto una mejora en la confianza entre evaluadores superior al $40 \%$, con una consecuencia en la convergencia de opiniones del 47 \% (Martínez-Falero, González-García, García-Abril y Ayuga-Téllez, 2018).

\section{NUEVA ESTRATEGIA PARA DECISIONES CONJUNTAS}

La importancia de conocer la evolución de la empatía en el tiempo es que sus variaciones se pueden incorporar a los procesos de gestión. Así, cuando a lo largo del proceso participativo ocurriera que al aumentar el número de participantes no se produjera un aumento de la empatía, entonces, el responsable final de la 
toma de decisiones debería dar por concluido el proceso de participación. Sin embargo, si al aumentar el número de participantes, también aumentara la empatía total, entonces, el proceso participativo debería estimularse con el fin de lograr una mayor convergencia entre preferencias empáticas.

Si en el momento de detener la participación, la convergencia de empatías fuera alta, entonces la opinión agregada sería representativa de las preferencias de las personas involucradas, y este valor debería ser el adoptado como la decisión conjunta del grupo. En caso contrario será necesario desagregar el conjunto de evaluadores en subgrupos homogéneos con una opinión común y aplicar otros procedimientos de agregación de preferencias.

Desde el punto de vista operativo, la nueva estrategia supone lo siguiente:

- Apertura de un proceso de información y participación online para que cualquier miembro empoderado de la organización formule sus preferencias.

- Fomento de la participación con community managers que animen la participación recurrente a los individuos ya implicados en el proceso (por si modificaran sus preferencias como resultado de interaccionar con otros participantes) y que movilicen la participación de otros a través de las redes sociales existentes.

- Representación de las opiniones de cada participante: supone determinar la forma en que cada participante valora cualquier solución (a partir de sus preferencias personales) y el diseño de la solución que mejor se adapta a cada sistema de preferencias individual.

- Cálculo online de la empatía de cada individuo con los restantes implicados en las decisiones y de la empatía total a partir de las opiniones de todos los participantes.

- Mantenimiento del proceso participativo mientras se produzcan aumentos de la empatía total y cancelación del proceso participativo cuando no se produzcan incrementos significativos de la empatía entre participantes.

- Aplicación, en el momento de cancelación, de las herramientas de la Web 2.0 y de la construcción de funciones interpersonales de utilidad para elegir la mejor decisión agregada o seleccionar soluciones representativas y socialmente aceptables para someterlas a algún proceso de elección social o individual.

Además de medir e incorporar la evolución de la confianza a los procesos de tomas de decisiones, la nueva estrategia posee otras características deseables en las decisiones conjuntas: satisface los requerimientos exigibles en los procesos de participación pública ${ }^{14}$, su desarrollo requiere implementar procesos de evaluación y de diseño de las mejores soluciones que, por sí mismos, suponen resultados valiosos en las decisiones conjuntas; además, la gestión de la confianza se integra en los sistemas digitales de gestión (obtiene la información necesaria de los sistemas de información de la organización, procesa la información con las herramientas de big-data y business analytic disponibles $y$, después de aplicar sus propios algoritmos, transfiere los resultados a los sistemas automáticos de apoyo a las decisiones que use la organización).

La estrategia propuesta aporta otro beneficio añadido: cuando se aplica en organizaciones sin ánimo de lucro se contrapone a los excesos de los nuevos desarrollos de las TIC que tienden a limitar el acceso universal a la información y a proponer decisiones que solo son económicamente eficientes. Al reforzar el alcance y la autoorganización, se potencia la igualdad de oportunidades y la responsabilidad personal, y al fomentar la convergencia de preferencias individuales, se apoya la consecución de soluciones socialmente aceptables.

Los algoritmos para la gestión de la confianza miden objetivamente la evolución de la confianza (véanse epígrafes 4.1 y 4.2 ) e incorporan estas mediciones en las decisiones de una organización. Estas decisiones mejoran simultáneamente los procesos a los que se aplican y la confianza general de la organización.

\footnotetext{
14 Básicamente: ser transparente y refutable por terceros en todas sus valoraciones; adaptable a la información disponible y al conocimiento que posee el evaluador en el proceso de participación; ser de acceso universal (su uso no se ve afectado por la brecha tecnológica, cultural o generacional); maximizador de la utilidad individual y socialmente inteligente y servir al promotor del proceso como instrumento para rendimiento de cuentas a la sociedad. Estos requisitos no están presentes conjuntamente en ninguna de las aplicaciones informáticas actuales (véanse epígrafes 2.1 a 2.3).
} 


\section{INCORPORACIÓN DE LA GESTIÓN DE LA CONFIANZA EN LAS ORGANIZACIONES}

Las expectativas de la gestión de confianza son muy altas, pero su implementación requiere más que la simple incorporación de nuevos algoritmos. Con objeto de que las mejoras inducidas por la confianza sean sostenibles para toda la sociedad es necesario incrementar todos los niveles relacionados con la seguridad (sobre todo, jurídica) y la fluidez del mercado. En España, sigue siendo urgente reforzar el acceso universal e informado a los resultados de la innovación, fomentar que las personas asuman riesgos y asegurar la propiedad de los beneficios de aquellos que han asumido los riesgos.

Pero, aunque fueran muchas las organizaciones que adoptaran la gestión basada en la confianza, la simple mejora en procesos o productos no crea ventajas competitivas -porque todas las organizaciones pueden alcanzan la misma eficiencia-.

Para que la gestión de la confianza aporte mejoras competitivas, las organizaciones necesitan un acceso privilegiado a los datos y poner en marcha una estructura organizativa flexible que facilite la incorporación de los cambios culturales que genera la confianza, tanto en la organización como en las personas que la forman. Algunos de estos cambios se comentan a continuación.

\subsection{Cambios para potenciar la interconexión entre datos y algoritmos}

Un primer cambio supone comprender que la interdependencia entre datos y algoritmos es crítica. Al igual que sucede con la IA, los algoritmos para la gestión de la confianza no son inteligentes per se: aprenden inductivamente analizando los datos de la organización (Ransbotham, Kiron, Gerbert y Reeves, 2017). En consecuencia, es indispensable entrenar a los algoritmos en los datos que se precisen en cada organización.

El entrenamiento de algoritmos va más allá de las actuaciones que se derivan de la decisión entre la compra y el desarrollo propio de tecnología. Independientemente de que algunas tareas se puedan externalizar $y$ de que los algoritmos se puedan comprar, es nece-

\author{
Para que la gestión de la confianza \\ aporte mejoras competitivas, las \\ organizaciones necesitan un acceso \\ privilegiado a los datos y poner en \\ marcha una estructura organizativa \\ flexible que facilite la incorporación \\ de los cambios culturales que genera \\ la confianza, tanto en la organización \\ como en las personas que la forman
}

sario incorporar a la organización personas capaces de comprender cómo se han construido los algoritmos, cómo hay que recopilar e integrar los datos necesarios para el entrenamiento de los algoritmos en esos datos y cómo supervisar este entrenamiento.

Pero los algoritmos no funcionan sin buenos datos; de hecho, la recopilación y la preparación de datos son actividades capitales en toda organización, aunque laboriosas. En realidad, todos los sistemas actuales de gestión requieren una estructura de información sólida que facilite el acceso a los datos pertinentes. Los datos externos se pueden comprar o conseguir mediante alianzas con otras organizaciones (empresas de medios, de telecomunicaciones, etc.), pero son los datos propios de cada organización los que marcan las ventajas competitivas en la gestión basada en la confianza (especialmente, los datos referentes al historial de las decisiones tomadas y la información de cómo actúan los individuos empoderados en sus decisiones) ${ }^{15}$.

\footnotetext{
15 La importancia de los datos propios se justifica por las inversiones previstas para acceder y organizar este tipo de información. A título de ejemplo, se menciona el número de dispositivos de IOT (internet of things) que se prevé que estén instalados en 2020 para la captura de información en las empresas y el montante total de las inversiones aprobadas para 2017 en tecnologías para análisis de la información disponible (bigdata y business analytic). Ambos datos están disponibles en la web.
} 


\subsection{Cambios organizativos y de gestión}

Algunos de los cambios necesarios inciden en la forma en que se debe organizar la entidad: empoderamiento de sus miembros, incorporación de personas que se fíen de los demás y flexibilización organizativa (para primar el conocimiento que se pone a disposición de otros y para fomentar nuevas formas de colaboración, que incluyen equipos de proyectos compuestos de seres humanos y máquinas). Otros cambios son aún más profundos porque afectan al comportamiento de cada persona.

Por la magnitud de los cambios necesarios (la experimentación y el aprendizaje), la gestión de la confianza puede llevar más tiempo que otras iniciativas digitales. Además, los cambios inducen una mayor vulnerabilidad al fracaso, pero también al éxito. En consecuencia, las organizaciones necesitan prepararse para una mayor incertidumbre, y esto afecta a la priorización de proyectos e inversiones.

Respecto a los sistemas de gestión, la incorporación de la confianza requiere sistemas organizacionales híbridos (centralizados y distribuidos) que den poder a los individuos en los asuntos en los que son expertos y que preserven los objetivos, los procedimientos principales y los sistemas de control sujetos a la política general de la organización.

\subsection{Cambios para integrar la gestión de la confianza y otros sistemas digitales}

Los costes de incorporar la gestión basada en la confianza se pueden reducir si las organizaciones emplean

\footnotetext{
.......

Pero las organizaciones se enfrentan a problemas para gestionar sus propios datos: en ocasiones, los datos generados por las propias organizaciones son propiedad de las personas que los han generado y no de la organización; otras veces, las leyes de protección de datos impiden el uso de los mismos (por ejemplo, en lo referente a sexo, raza o religión); adicionalmente, los organismos reguladores pueden exigir la transparencia del proceso de toma de decisiones y obligar a la publicación de los datos 0 a realizar un informe tan detallado de los procesos de integración de la información que anula las ventajas competitivas; también es de destacar que la información puede estar muy fragmentada y suele estar poco estructurada (mucha información esencial está en forma de textos); finalmente -aunque estas no son las únicas dificultades-, se menciona la carencia generalizada en las organizaciones de datos negativos, pero los algoritmos progresan más con información de procesos que han fallado que con información relativa a los éxitos obtenidos. Todo esto convierte el análisis de la información en un proceso estratégico para muchas organizaciones.
}

\author{
Los costes de incorporar la gestión \\ basada en la confianza se pueden \\ reducir si las organizaciones emplean \\ los recursos utilizados para activar \\ otros sistemas de gestión digital, \\ específicamente para incorporar la IA
}

los recursos utilizados para activar otros sistemas de gestión digital, específicamente para incorporar la IA.

Es evidente que existen diferencias entre la IA y la gestión de la confianza: la gestión de la confianza utiliza algoritmos propios (algoritmos para la representación de la opinión de cada persona, para medir la evolución de la empatía global a lo largo del proceso participativo y para la agregación de opiniones) que no se inscriben en las principales tecnologías de IA (machine learning, procesamiento del lenguaje natural y robótica). Además, en la gestión basada en la confianza, la fuente principal de información es la experiencia personal de los individuos empoderados y en la IA es el historial de decisiones de la organización. Finalmente, la gestión de la confianza requiere cambios en la organización y el desarrollo de procesos que no necesitan la IA (el empoderamiento de los miembros de la organización, la incorporación de personas que se fíen de los demás y que la confianza que generen esas personas se transmita al resto de la organización).

No obstante, hay similitudes que favorecen las sinergias entre ambos sistemas de gestión: ambos requieren procesos muy intensos en datos -pueden llegar a hacer girar la competitividad de la organización en torno al acceso a la información requerida para sus procesos-; es imprescindible el entrenamiento de los algoritmos incorporados con datos de la propia organización y, en ambos casos, hay que adoptar una estrategia de gestión flexible. Especialmente hay que destacar las sinergias que se derivan de la necesidad de contar con una correcta identificación de las tipologías de las decisiones en cada organización.

Para caracterizar las decisiones, las tecnologías de IA suelen combinar los datos disponibles con programas de emparejamiento difuso y de autoaprendizaje para identificar patrones de problemas (tipos de pro- 
blemas y su solución histórica en la organización). La gestión basada en la confianza añade, a cada patrón, la representación de las opiniones de todas las personas empoderadas para su resolución e incorpora toda esta información en algoritmos propios de toma de decisiones. En la actualidad, la IA proporciona herramientas eficaces para la identificación de tipos de decisiones ${ }^{16}$; sus soluciones son mejores que las que aportan otros tipos de análisis para identificar tags (como el root-cause analysis) o las taxonomías y ontologías clásicas.

\section{DISCUSIÓN Y CONCLUSIONES}

La metodología descrita en el presente artículo propone procedimientos para medir la evolución de la confianza en organizaciones empoderadas y relacionar este concepto con la toma de decisiones. La metodología es compatible con el hecho de permitir que cada miembro participe tanto en los beneficios que proporciona a la organización como de los riesgos que asume.

Como todos los modelos de digitalización, la gestión basada en la confianza es muy dependiente de la información disponible. Actualmente, el volumen de datos es enorme y el acceso a las fuentes de información es un proceso complejo. La identificación, obtención y digitalización de estos datos se ha convertido en uno de los procesos principales de las organizaciones. Además, para la gestión de la confianza, la interdependencia entre datos y algorítmica requiere un importante periodo de aprendizaje y adaptaciones mutuas.

En estas condiciones -y con la metodología verificada, aunque no validada-, la decisión de incorporar la gestión basada en la confianza se debe tomar en el en-

\footnotetext{
16 Una historia de éxito en este sentido la proporciona Airbus. Para acelerar la producción del A350, Airbus necesitaba responder rápidamente a las interrupciones en la fabricación y se volvió hacia la IA: combinó datos de programas de producción de anteriores aviones con los del A350 y con algoritmos de IA e identificó los principales patrones de los problemas de producción. Los algoritmos reconocen el problema y proporcionan al operario una recomendación inmediata (el problema puede ser nuevo para una persona, pero ha podido ocurrir algo similar en las líneas de producción del A350, de otros programas o incluso en contextos diferentes). Esto ha permitido a Airbus acortar el tiempo de interrupciones en más de un tercio.
}

torno de la teoría de juegos. Una buena estrategia sería aceptar probabilidades altas de que se produzcan consecuencias negativas limitadas, junto con probabilidades menores de que se produzcan sucesos con consecuencias muy positivas. Si las pérdidas, aunque probables, están acotadas y son asumibles por la organización, la ocurrencia del suceso positivo -aunque su probabilidad de ocurrencia no se pueda medir por ser un suceso raro e inédito hasta el momento- marcará el futuro de la organización. En nuestro caso, en una organización flexible y con robustos sistemas de información, los costes de incorporar y entrenar los algoritmos de gestión de la confianza son limitados y, por el contrario, los beneficios que puede producir la gestión basada en la confianza pueden suponer ventajas definitivas. Las organizaciones no tan adaptadas a la era digital pueden adoptar la misma estrategia y desarrollar aplicaciones piloto que les ayuden a estar posicionadas ante una posible aceleración en la incorporación de este tipo de gestión.

Los posibles campos de aplicación son múltiples. Como ejemplo se pueden citar los siguientes:

- La negociación para la resolución de conflictos de todo tipo: negociación de conflictos laborales, acuerdos reguladores, crisis en la gestión de emergencias, etc.

- La mejora en la gestión de grupos de trabajo, facilitando la optimización de los recursos de cara a lograr objetivos precisos con las restricciones estimadas, como pudieran ser el tiempo de resolución, indicadores de crecimiento, sostenibilidad, integración con otros colectivos u objetivos, etc.

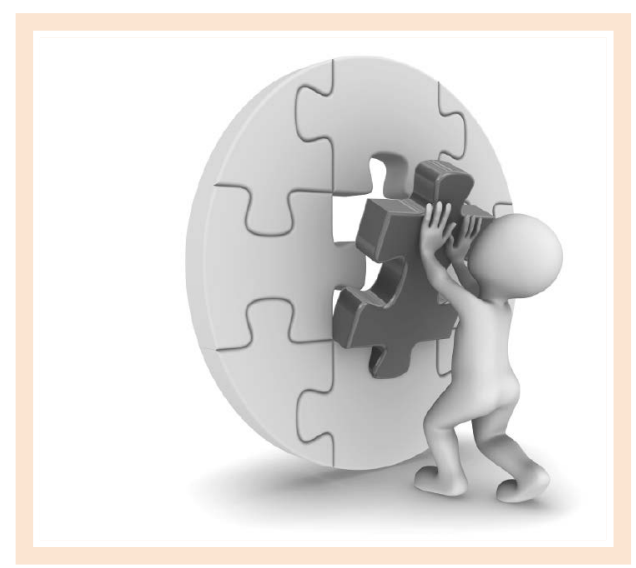




\section{BIBLIOGRAFÍA}

Adler, M. D. (2014). Extended preferences and interpersonal comparisons: a new account. Economics and Philosophy, 30, 123-162.

Aleskerov, F., Bouyssou, D. y Monjardet, B. (2007). Utility Maximization, Choice and Preference. Springer. (2. ${ }^{a}$ ed.). Berlin: Springer (Studies in Economic Theory, vol. 16).

Arrow, K. (1959). A difficulty in the concept of social welfare. Journal of Political Economy, 58(4), 328-346.

Arrow, K. (1963). Social Choice and Individual Values. (2. ${ }^{a}$ ed.). EE. UU.: Yale University Press.

Arrow, K. (1978). Extended sympathy and the problem of social choice. Philosophia, 7, 233-237.

Ayuga-Téllez, E., González-García, C. y Martínez-Falero, E. (2013). Multiparticipant decision-making. En E. Martínez-Falero, S. Martín-Fernández y A. GarcíaAbril (Eds.), Quantitative Techniques in Participatory Forest Management (pp. 499-556). Boca Ratón, Florida, EE. UU.: CRC Press.

Bentham, J. (1789). An Introduction to The Principles of Moral and Legislation. London: Payne.

Binmore, K. (2005). Natural Justice. Oxford University Press.

Bonabeau, E. (2009). Decisions 2.0: the power of collective intelligence. MIT Sloan Management Review, 50(2), 45-52.

Borda, J. C. (1784). Mémoire sur les élections au scrutin. Histoire de l'Académie Royale des Sciences (Année 1781, pp. 657-665). Paris, France.

Condorcet, M. (1785). Essai sur l'application de l'analyse 'a la probabilité des décisius rendues á la pluralité des voix. Paris, France.

Debreu, G. (1954). Representation of a preference ordering by a numerical function. En R. Thrall, C. H. Coombs y R. Davies (Eds.), Decision Processes (pp. 159-175). New York: Wiley.

Dionne, S. D., Sayama, H., Hao, C. y Bush, B. J. (2010). The role of leadership in shared mental model convergence and team performance improvement: an agent-based computational model. The Leadership Quarterly, 21(6), 1.035-1.049.
Edgeworth, F. J. (1881). Mathematical psychics: an easy on the application of mathematics to moral sciences. London: Kegal Paul.

Grönlund, A., Holme, P. y Minnhagen, P. (2007). Dynamic scaling regimes of collective decision making. Europhysics Letters, 81(2), 1-6. doi: 10.1209/0295-507 5/81/28003.

Hammond, P. J. (1991). Interpersonal comparisons of utility: why and how they are and should be made. En J. Elster y J. E. Roemer (Eds.), Interpersonal Comparisons of Well-Being. Cambridge: Cambridge University Pres.

Hardin, R. (1988). Morality within the Limits of Reason. Chicago: The University of Chicago Press.

Harsanyi, J. (1955). Cardinal welfare, individualistic ethics, and the interpersonal comparison of utility. Journal of Political Economy, 63, 309-321.

Harsanyi, J. (1977). Rational Behavior and Bargaining Equilibrium in Games and Social Situations. Cambridge: Cambridge University Press.

Harsanyi, J. (1992). Normative validity and meaning of Von Neumann and Morgenstern utilities. En B. Skyrms (Ed.), Studies in Logic and the Foundations of Game Theory: Proceedings of the Ninth International Congress of Logic, Methodology and the Philosophy of Science. Dordrecht: Kluwer.

Hume, D. (1739). A Treatise of Human Nature. L. A. Selby-Bigge [Ed.] y P. Nidditch [Rev.]. (2. ${ }^{a}$ ed. 1978). Oxford: Clarendon Press.

Layard, R. (2005). Happiness: Lessons from a New Science. London: Allen Lane.

Liu, P. y Teng, F. (2016). An extended TODIM method for multiple attribute group decision-making based on 2-dimension uncertain linguistic variable. Complexity, 21(5), 20-30.

Marshall, A. (1890). Principles of economics. (9. ${ }^{a}$ ed. 1961). London: Macmillan.

Martínez-Falero, E., Martín-Fernández, S. y Orol, S. (2013). Assessment of sustainability based on individual preferences. En E. Martínez-Falero, S. Martín-Fernández y A. García-Abril (Eds.), Quantitative Techniques in Participatory Forest Management (pp. 367-420). Boca Ratón, Florida, EE. UU.: CRC Press. 
Martínez-Falero, E., Ayuga-Téllez, E., González-García, C., Grande-Ortiz, M. ${ }^{a}$ Á. y Sánchez de Medina Garrido, Á. (2017). Experts' analysis of the quality and usability of SILVANET software for informing sustainable forest management. Sustainability, 9, pp. 1-13. doi: 10.3390/su9071200.

Martínez-Falero, E., González-García, C., García-Abril, A. y Ayuga-Téllez, E. (2018). Participatory forest managemnet based on confidence. Forest (artículo invitado en prensa).

Martín-Fernández, S., Martínez-Falero, E. y ValentínGamazo, M. (2013). Optimization methods to identify the best management plan. En E. Martínez-Falero, S. Martín-Fernández y A. García-Abril (Eds.), Quantitative Techniques in Participatory Forest Management (pp. 421-498). Boca Ratón, Florida, EE. UU.: CRC Press.

Martín-Fernández, S. y Martínez-Falero, E. (2018). Sustainability assessment in forest management based on individual preferences. Journal of Environmental Management, 206, 482-489.

McHugha, K. A., Yammarino, F. J., Dionne, S. D., Serban, A., Sayama, H. y Chatterjee, S. (2016). Collective decision making, leadership, and collective intelligence: tests with agent-based simulations and a field study. The Leadership Quarterly, 27, 218-241.

Myers, D. G. (2002). Intuition: Its Powers and Perils. New Haven, CT: Yale University Press.

Neumann, J. von y Morgenstern, O. (1944). The Theory of Games and Economic Behavior. Princeton: Princeton University Press.

Oztürk, M., Tsoukiàs, A. y Vincke, P. (2005). Preference modelling. En J. Figueira, S. Greco y M. Ehrgott (Eds.), Multiple Criteria Decision Analysis: State of the Art Surveys (pp. 27-72). Boston, Dordrecht, London: Springer Verlag.

Page, K. M. y Martin, A. N. (2002). Empathy leads to fairness. Bulletin of Mathematical Biology, 64(6), 1.101-1.116.

Ransbotham, S., Kiron, D., Gerbert, P. y Reeves, M. (September 6, 2017). Reshaping business with artificial intelligence [reseach report]. MIT Sloan Management Review.

Rawls, J. (1971). A Theory of Justice. Oxford: Oxford University Press.
Robbins, L. (1938). Interpersonal comparison of utility: a comment. Economic Journal, 47(2), 635-641.

Rodríguez, M.A., Steinbock, D. J., Watkins, J. H., Gershenson, C., Bollen, J., Grey, V. y deGraf, D. (2007). Smartocracy: social networks for collective decision making. Proceedings 40th Hawaii International Conference on Systems Science, 3-6 January 2007 (pp. 90-100). Waikoloa, Big Island, HI, EE. UU.: IEEE Computer Society.

Rovarini, P., Cerviño, M. y Juárez, G. (2009). Globalización: necesidad de toma de decisiones colectivas. Primer Congreso Boliviano de Ingeniería y Tecnología IEEEUMSE. La Paz, Bolivia: Universidad Mayor de San Andrés.

Salehi-Abari, A. y Boutilier, C. (2014). Empathetic social choice on social networks. En A. Lomuscio, P. Scerri, A. Bazzan y M. Huhns (Eds.). Proceedings of the 13th International Conference on Autonomous Agents and Multiagent Systems (AAMAS 2014), May 5-9 2014. Paris, France.

Sayama, H., Farrell, D. L. y Dionne, S. D. (2011). The effects of mental model formation on group decision making: an agent-based simulation. Complexity, 16(3), 49-57.

Sen, A. (1970). Collective Choice and Social Welfare. San Francisco: Holden Day.

Sen, A. (1976). Welfare inequalities and rawlsian axiomatics. Theory and Decision, 7, 243-262.

Sen, A. (1978). Rational behaviour. En J. Eatwell, M. Milgate y P. Newman. (Eds.), The New Palgrave. London: Macmillan.

Sen, A. (1998). The possibility of social choice. Economic Sciences, 178-206. Nobel lecture at Trinity College, Cambridge, CB2 1TQ, Great Britain, on December 8.

Smith, A. (1759). The Theory of Moral Sentiments. Oxford: Clarendon Press.

Stensholt, E. (2011). Voces populi and the art of listening. Social Choice and Welfare, 35(2), 291-317.

Suppes, P. (1966). Some formal models of grading principles. Synthèse, 6, 284-306.

Suzumura, K. (1983). Rational Choice, Collective Decisions and Social Welfare. Cambridge: Cambridge University Press.

Taleb, N. N. (2012). Antifragile: Things that Gain from Disorder. New York: Random House Trade Paperbacks. 